\title{
Evidências de Validade da Versão Brasileira da Escala de Workaholism (DUWAS-16) e sua versão breve (DUWAS-10)
}

\author{
Ana Claudia Souza Vazquez ${ }^{1}$ \\ Universidade Federal de Ciências da Saúde de Porto Alegre, Porto Alegre-RS, Brasil \\ Clarissa Pinto Pizarro de Freitas \\ Universidade Salgado de Oliveira - UNIVERSO, Niterói-RJ, Brasil \\ Andressa Cyrre \\ Universidade Federal de Ciências da Saúde de Porto Alegre, Porto Alegre-RS, Brasil \\ Claudio Simon Hutz \\ Universidade Federal do Rio Grande do Sul, Porto Alegre-RS, Brasil \\ Wilmar B. Schaufeli \\ Utrecht University \& Leuven University, Leuven, Bélgica
}

\section{RESUMO}

O workaholism caracteriza-se por um estado mental em que os profissionais trabalham excessiva e compulsivamente. Este estudo objetivou avaliar as propriedades psicométricas das versões extensa (DUWAS-16) e reduzida (DUWAS-10) da Dutch Work Addiction Scale (DUWAS) para o contexto brasileiro. Participaram da pesquisa 571 trabalhadores $(65,5 \%$ do sexo feminino) com idade média de 36,4 anos (DP = 10,8). A análise fatorial confirmatória (AFC) da DUWAS-16 evidenciou que a escala pode ser utilizada adotando uma estrutura de dois fatores oblíquos (Trabalhar Excessivamente e Trabalhar Compulsivamente) ou uma estrutura unidimensional (Adição). A AFC da versão reduzida DUWAS-10 sugere que tanto a estrutura unidimensional (Adição) ou de dois fatores oblíquos (Trabalhar Excessivamente e Trabalhar Compulsivamente) são adequadas. Os resultados indicaram que a DUWAS-10 pode ser considerada superior a DUWAS-16 para avaliar as dimensões de workaholism no contexto brasileiro.

Palavras-chave: adição ao trabalho; evidências de validade; avaliação psicológica.

\section{ABSTRACT - Validity evidence of the Dutch Work Addiction Scale - Brazilian Version}

Workaholism is characterized by a state of mind in which professionals work excessively and compulsively. This study aimed to evaluate the psychometric properties of two versions of the Dutch Work Addiction Scale (DUWAS-16 and DUWAS-10) for the Brazilian context. A total of 571 workers, $65.5 \%$ female, with a mean age of 36.4 years (SD = 10.8) participated in the study. The confirmatory factor analysis (CFA) of DUWAS-16 showed that the scale can be used by adopting a structure of two oblique factors (Working Excessively and Working Compulsively), or a one-dimensional structure (Addiction). The CFA of the DUWAS-10 reduced version suggests that either the one-dimensional structure (Addiction) or two oblique factors (Working Excessively and Working Compulsively) are adequate. The results indicated that DUWAS-10 can be considered superior to DUWAS-16 to evaluate the dimensions of workaholism in the Brazilian context.

Keywords: addiction to work; validity evidence; psychological evaluation.

RESUMEN - Evidencias de Validez de la Versión Brasileña de la Escala de Workaholism (Adicción al Trabajo) DUWAS El workaholism se caracteriza por un estado mental en que los profesionales trabajan de forma excesiva y compulsiva. Este estudio tuvo por objetivo evaluar las propiedades psicométricas de la versión extensa (DUWAS-16) y la versión reducida (DUWAS-10) de la Dutch Work Addiction Scale (DUWAS) en el contexto brasileño. Los participantes de la investigación fueron 571 trabajadores (65,5\% de sexo femenino) con edad promedio de 36.4 años (DP = 10.8). El análisis factorial confirmatorio (AFC) de la DUWAS-16 evidenció que la escala puede ser utilizada adoptando una estructura de dos factores oblicuos (Trabajar Excesivamente y Trabajar Compulsivamente), o una estructura unidimensional (Adicción). La AFC de la versión reducida DUWAS-10 señaló que tanto la estructura unidimensional (Adicción) como la de dos factores oblicuos (Trabajar Excesivamente y Trabajar Compulsivamente) son adecuadas. Los resultados indicaron que la DUWAS-10 puede ser considerada superior a la DUWAS-16 para evaluar las dimensiones de workaholism en el contexto brasileño.

Palabras-clave: adicción al trabajo; evidencias de validez; evaluación psicológica. 
Apesar da ampla utilização do termo "adição", um dos desafios atuais para os pesquisadores são os poucos estudos sobre as suas causas, consequências e consenso conceitual (Mazzetti, Biolcati, Guglielmi, Vallesi, \& Schaufeli, 2016; Hu et al., 2014). O objetivo desta pesquisa foi adaptar as versões de 16 itens e 10 itens da Dutch Work Addiction Scale (DUWAS) para o contexto brasileiro e investigar o funcionamento da dimensão Percepção de Trabalho Excessivo nesse contexto, com a finalidade de contribuir para avanços nesse campo de conhecimento.

O crescente interesse na adição ao trabalho ou workaholism é impulsionado por elevadas demandas no processo laboral que visam conduzir as práticas profissionais para aumento constante da competitividade nas organizações. Tais exigências conduzem as pessoas a trabalhar por mais horas e a ter um intenso e contínuo envolvimento com a organização para se adaptar e reagir às necessidades diárias do trabalho. Nesse contexto, a adição é caracterizada pelo trabalho excessivo associado à necessidade interna de trabalhar constantemente (Schaufeli, Taris, \& Bakker, 2008).

$\mathrm{Na}$ patologia do workaholism, que se desenvolve de modo processual, o indivíduo perde gradativamente o controle emocional sobre suas atitudes pela compulsão de realizar elevada quantidade de tarefas e ser bem-sucedido. Os adictos ao trabalho extrapolam suas atividades laborais para contextos extralaborais, o que afeta sua vida como um todo de modo mais negativo do que prazeroso (Carlotto, Wendt, Lisboa, \& Moraes, 2014; Schaufeli et al., 2008; Killinger, 1991). Estudos que contribuam para aprofundar a compreensão sobre esse fenômeno se justificam pelo foco no bem-estar e em intervenções positivas na organização do trabalho.

\section{Workaholism}

A adição no trabalho é uma dependência laboral em que o indivíduo sente compulsão para trabalhar excessivamente e obter êxito constante em suas atividades profissionais (Carlotto, 2011; Mazzetti et al., 2016). Tais exigências não são o resultado direto de demandas laborais, nem advém do prazer intrínseco em trabalhar. O processo da adição ao trabalho é promovido por pressão interna do próprio trabalhador, fator que o aflige e faz com que se sinta culpado sempre que não está trabalhando ou realizando algo que considera produtivo.

Adictos trabalham por mais tempo e mais arduamente do que os outros profissionais porque eles tendem a criar, para si, altas exigências para com suas atividades laborais. Segundo Schaufeli, Taris e Bakker (2008), o que diferencia os adictos de trabalhadores engajados é o desejo obsessivo e fora de controle sobre o trabalho e as horas dedicadas a ele. A consequência mais imediata desse tipo de comportamento é a sobrecarga de trabalho, tanto para si como para colegas ou liderados, pela baixa percepção de que o trabalho está sendo realizado em demasia.
Por conta de sua compulsão, os trabalhadores adictos se envolvem em tarefas excessivas em comparação ao que lhes é demandado. À medida que a adição ao trabalho se confunde com o desejado alto desempenho nas organizações, aumenta o risco de exposição destes à sobrecarga laboral. Não obstante, pesquisas apontam que o workaholism tem impacto negativo para as organizações, pois seus resultados efetivos são menos produtivos na prática que o esperado (Shimazu, Schaufeli, Kamiyama, \& Kawakami, 2015; Mazzetti et al., 2016).

Evidências têm demonstrado que profissionais workaholics são extremamente envolvidos com o trabalho e se sentem intrinsecamente forçados ou atraídos a trabalhar (Mazetti, Schaufeli, \& Guglielmi, 2014; Shimazu et al., 2015). Já Sousa, Mónico e Castro (2012) argumentam que o trabalho excessivo pode reduzir o sofrimento do adicto, já que ele se sente compelido a trabalhar. Para esses autores, a base da obsessão pelo trabalho está relacionada a estímulos pessoais acoplados às demandas organizacionais que são relacionadas à satisfação de necessidades psicológicas por meio de atividades laborais, o trabalho, às recompensas financeiras ou ao status social obtido.

Por outro lado, Carlotto, Wendt, Lisboa e Moraes (2014) discutem a busca incessante dos adictos laborais em superar suas próprias expectativas, a qual extrapola os limites ou demandas da organização. Mesmo em situações nas quais eles não estão no ambiente laboral, não conseguem deixar de trabalhar. Essa elevada quantidade de trabalho vem acompanhada de elevados níveis de ansiedade, o que compromete a qualidade de suas atividades profissionais e afeta negativamente suas relações com seus colegas de trabalho. O impacto negativo do workaholism se dá tanto para o indivíduo, pelo desprazer que ele sente com o trabalho e pela sobrecarga de atividades, como para a organização (Shimazu et al., 2015; Mazzetti et al., 2016).

A Dutch Work Addiction Scale (DUWAS) foi elaborada como instrumento de avaliação da adição em trabalhadores. Pesquisas em vários países têm confirmado sua validade e características psicométricas satisfatórias (Hu et al., 2014). No Brasil, há carência de estudos científicos sobre essa adição no trabalho. Portanto, essa pesquisa buscou contribuir para avanços nessa temática pela adaptação e validação da versão brasileira da DUWAS e sua versão reduzida. Além disso, foi proposta a adaptação e a avaliação de evidências de validade da subescala Percepção de Trabalho Excessivo, a pedido de um dos autores originais da DUWAS que estava estudando-a como uma possível dimensão do workaholism.

\section{Método}

\section{Participantes}

Participaram 571 trabalhadores, $34,5 \%$ do sexo masculino e $65,5 \%$ do sexo feminino, com idades entre 19 e 74 anos $(M=36,7$ anos, $D P=11,8)$. A maioria $(90,7 \%)$ tinha nível superior completo ou em curso, sendo que 
$68,4 \%$ possuia pós-graduação $(n=359)$ e $26,4 \%$ tinham concluído o ensino técnico ou menos $(n=186)$. A amostra de conveniência foi composta de participantes das cinco regiões brasileiras, com $76,2 \%$ da Sul; $18,5 \%$ da Sudeste; $1,9 \%$ do Centro-Oeste; $1,8 \%$ do Nordeste; e $1,4 \%$ do Norte. Entre estes, 23\% trabalhavam mais de 44 horas semanais; $52,6 \%$ por 44 horas; $17 \%$ entre 20 a 30 horas e 7,3\% por menos de 20 horas. A média de renda familiar variou entre menos de um salário mínimo (SM) $(4,8 \%, n=25)$, um a dois a SM $(21,6 \%, n=113)$, três a quatro SM $(20,4 \%, n=107)$, cinco a seis SM $(16,8 \%$, $n=88)$ e sete ou mais SM $(36,5 \%, n=191)$. No momento da pesquisa, $62,3 \%$ trabalhadores possuíam função técnica; $11,4 \%$ de supervisão de equipes; $24 \%$ de direção e $23,5 \%$ de estagiário.

\section{Instrumento}

A DUWAS apresenta 16 itens, em duas dimensões: (1) Trabalhar Excessivamente (TE), mensurada nos itens 3, 4, 6, 8, 11, 13, 17; e (2) Trabalhar Compulsivamente (TC), nos itens $2,5,7,9,12,14,15,18,19$. São avaliados em uma escala tipo Likert de 1 (quase nunca) a 4 (quase sempre) pontos. Na versão original, a DUWAS apresenta com consistência interna satisfatória $(\alpha=0,80$ para TE e $\alpha=0,86$ para a TC) e a relação de TC e TE apresentou média magnitude $(r=0,75 ; p<0,001)$ (Schaufeli et al., 2008).

A dimensão Percepção de Trabalho em Demasia (PTD) foi proposta por um dos autores da DUWAS original, com base no estudo de Castillo e Gomez (2012), que demonstrou sua relação positiva com indicadores de horas gastas no trabalho e o comportamento de pensar no trabalho quando não está trabalhando. A PTD é composta pelos itens $1,10,16,20$, respondidos em uma escala tipo Likert de 1 (quase nunca) a 4 (quase sempre) pontos.

A DUWAS-10 foi proposta por Schaufeli, Shimazu e Taris (2009), a fim de investigar o workaholism e suas dimensões Trabalhar Excessivamente (TE, itens 3, 4, 6, 13, 17) e Trabalhar Compulsivamente (TC, itens 5, 9, $15,18,19)$ por meio de uma escala breve. Os participantes respondem aos itens em uma escala tipo Likert de 1 (quase nunca) a 4 (quase sempre) pontos. A versão original da DUWAS-10 apresentou índices de consistência interna satisfatórios (TE, $\alpha=0,78$ na amostra holandesa; $\alpha=0,73$ na amostra japonesa; TC, $\alpha=0,78$ na amostra holandesa e $\alpha=0,68$ na amostra japonesa), as correlações das dimensões TE e TC apresentaram magnitude média na amostra holandesa $(r=0,50 ; p<0,001)$ e japonesa $(r=0,59 ; p<0,001)$ (Schaufeli, Shimazu, \& Taris, 2009).

As pessoas são consideradas viciadas em trabalho quando os escores são iguais ou superiores ao percentil 75 na combinação entre TC + TE ou no escore de Adição ao Trabalho. A PTD, por sua vez, não contribui diretamente para a identificação do profissional como workaholic; mas é utilizada como uma dimensão que descreve a percepção do trabalhador sobre a sua carga de trabalho, mesmo que ele não seja um adicto ao trabalho. O escore das dimensões é obtido pela média da pontuação obtida em cada dimensão (TE, TC e PTD). Já os escores de workaholism são avaliados pela média dos itens das dimensões TC e TE.

\section{Procedimentos para tradução e validação de conteúdo}

A tradução foi feita por três juízes brasileiros, fluentes em inglês, com especialização em Psicometria ou em Psicologia Organizacional e do Trabalho. Todos possuíam experiência prévia em tradução de escalas e estavam cientes da importância de manter a semelhança conceitual dos itens no processo, a fim de maximizar a equivalência entre o original e a versão brasileira (Pacico, 2015). A convergência dos resultados gerou a primeira versão em português. Ajustes foram feitos pelos revisores para deixar as frases mais próximos ao português brasileiro, conforme as normas metodológicas internacionais para adequação de instrumentos para outras culturas (AERA, APA, \& NCME, 2014).

A validade de conteúdo foi avaliada em três grupos focais, por nível de escolaridade: profissionais de ensino médio $(n=3)$; profissionais de ensino superior $(n=3)$ e profissionais de ensino fundamental $(n=3)$. Os participantes foram convidados a ler a escala, informar sua compreensão de cada item e relatar a necessidade de inclusão de novos fatores ou itens na escala. A coleta foi até o ponto da saturação teórica. Foram feitas adaptações na redação dos itens, porém não houve inclusão de novos itens ou dimensões na versão brasileira da escala.

Foi realizada a tradução reversa da versão adaptada por um pesquisador bilíngue com conhecimento do construto; a qual foi enviada para avaliação de um dos autores da versão original da DUWAS. Nesse processo, três itens foram ajustados para caracterizar melhor as dimensões do construto. O item 6 foi alterado de "Mantenho-me ocupado e muito produtivo no meu trabalho" para "Mantenho-me ocupado e realizo várias atividades simultaneamente no meu trabalho"; o item 11 de "Eu me coloco sob pressão por me impor prazos quando trabalho" para "Eu tenho a tendência de me colocar sob pressão por me impor prazos finais quando trabalho"; e o item 19 de "Para mim é difícil relaxar quando não estou trabalhando" para "Para mim é duro relaxar quando não estou trabalhando". O processo de tradução e retroanálise das semelhanças entre o original e a versão brasileira forneceu dados importantes de equivalência, sendo fundamental, nessa etapa, a participação do autor do teste.

\section{Procedimento coleta de dados}

A coleta de dados para a versão brasileira da DUWAS foi obtida por meio de pesquisa on-line em redes sociais dos pesquisadores pela ferramenta SurveyMonkey, entre 
agosto e setembro de 2015. Todos os participantes foram informados sobre os objetivos da pesquisa e aqueles que concordaram com o termo de consentimento livre esclarecido obtiveram acesso aos instrumentos da pesquisa.

\section{Procedimentos para análise e interpretação dos dados}

Para definir a estrutura fatorial da versão brasileira da DUWAS -16, DUWAS-16 + PTD e DUWAS-10, foram aplicados dois critérios de extração: os pressupostos teóricos (adição ao trabalho constitui-se por três dimensões, e adição ao trabalho constitui-se por duas dimensões) e a Análise Paralela (Hayton, Allen, \& Scarpello, 2004). A decisão pelo número de fatores a serem retidos foi realizada com base nos resultados desses critérios, de modo que dois modelos foram testados para avaliar a DUWAS-16 + PTD, DUWAS-16 e a DUWAS-10. Foi utilizado o método de extração Weighted Least Squares Mean and Variance-Adjusted (WLSMV) com rotação oblíqua (geomin), por esse ser um método de extração robusto para dados ordinais (Muthén \& Muthén, 2010). Os índices de ajuste foram os seguintes: CFI $(>0,90)$, TLI $(>0.90)$, e RMSEA $(<0,08$, com intervalo de confiança de $90 \%$ não excedendo a 0,10 ) (Brown, 2006). Os índices de modificação (IM) com valores acima de 50,00 foram avaliados, para identificar fontes de problemas na especificação do modelo (Brown, 2006).

O primeiro modelo da DUWAS-16 + PTD avaliou os índices de ajuste da estrutura unifatorial; na qual os 20 itens carregam em um fator geral de adição ao trabalho. O segundo modelo investigou os índices de ajuste da solução de três fatores de primeira ordem para adição ao trabalho (Trabalhar Excessivamente, Trabalhar Compulsivamente e Percepção de Trabalho em Demasia) (Castillo \& Gomez, 2012).

A análise da DUWAS-16 foi realizada investigando primeiro se essa escala apresentaria uma estrutura unifatorial, com os itens de TE e TC carregando no mesmo fator. Após essa avaliação, foram investigados os índices de ajuste da solução de dois fatores de primeira ordem para adição ao trabalho (Trabalhar Excessivamente e Trabalhar Compulsivamente) (Schaufeli et al., 2009).

Os modelos testados para a DUWAS-10 foram o unifatorial (todos os itens carregavam em um fator geral) e o de Dois Fatores de Primeira Ordem (Trabalhar Excessivamente e Trabalhar Compulsivamente), conforme proposto por Schaufeli et al. (2009). A dimensão Percepção de Trabalho em Demasia não é avaliada no DUWAS-10, pois a versão reduzida foca-se em investigar as dimensões Trabalhar Excessivamente e Trabalhar Compulsivamente, dimensões centrais do workaholism (Schaufeli et al., 2009).

Tendo em vista a importância do uso de instrumentos breves em pesquisas, foi investigado se há equivalência da mensuração do traço latente das dimensões entre a
DUWAS-20 e a DUWAS-10. Foi calculado o tamanho de efeito $\left(r^{2}\right)$ das correlações de zero-ordem entre os pares de dimensões, sendo as correlações testadas utilizando seus escores fatoriais na modelagem de equações estruturais. Os índices de consistência interna foram avaliados por meio do coeficiente de fidedignidade composta $(f c)$.

Foram investigadas as relações entre os índices de Trabalhar Compulsivamente, Trabalhar Excessivamente e adição no trabalho como um construto unifatorial com as características sociodemográficas (sexo) e laborais (escolaridade e função). Foram realizadas análises de teste t para verificar as diferenças nos índices das dimensões de adição ao trabalho por sexo (feminino e masculino), escolaridade (técnicos e especialistas) e função laboral (colaboradores e gestores). Além disso, foi calculado o tamanho de efeito $d$ de Cohen para investigar a importância prática desses resultados.

Pontua-se que, devido ao expressivo tamanho da amostra, o nível de significância de todas as análises inferenciais (equações estruturais e comparação de média) foi estabelecido em $0,001(p<0,001)$. Esse procedimento foi adotado a fim de evitar a presença de Erro Tipo I, ou seja, encontrar diferenças estatisticamente significativas segundo o valor de $p$, mas com baixo tamanho de efeito e relevância prática espúria.

A identificação dos profissionais que estão vivenciando workaholism é realizada por meio da análise da pontuação dos escores nas dimensões Trabalhar Compulsivamente e Trabalhar Excessivamente, ou por meio da análise do escore geral de adição ao trabalho. Os profissionais são reconhecidos como workaholics nas situações em que seus escores nas duas dimensões Trabalhar Compulsivamente e Trabalhar Excessivamente é superior ao percentil 75. Dessa forma, os escores da dimensão Trabalhar Compulsivamente e Trabalhar Excessivamente avaliados pela escala DUWAS-16 devem ser superiores a 2,3 e a 2,9. Ao utilizar a DUWAS-10, os escores das dimensões Trabalhar Compulsivamente e Trabalhar Excessivamente devem ser superiores a 2,2 e a 2,8. Outra forma de identificar os escores de workaholism é por meio do escore de adição ao trabalho, dessa forma, ao aplicar a escala DUWAS-16 ou DUWAS-10, participantes com escores superiores a 2,5 serão identificados como workaholics.

\section{Resultados}

O método de análise paralela demonstrou que a estrutura unidimensional seria a mais adequada para a DUWAS-16 + PTD. Não obstante, os pressupostos teóricos sugeriram a retenção de três fatores de primeira ordem (Castillo \& Gomez, 2012). Por isso, foram testados dois modelos para a estrutura da DUWAS-16 + PTD. O Modelo 1 avaliou a estrutura unidimensional, com os 20 itens carregando em um fator geral de adição ao trabalho. O Modelo 2 investigou a estrutura de três 
fatores de primeira ordem, no qual os itens carregaram nas dimensões Trabalhar Compulsivamente, Trabalhar Excessivamente e Percepção de Trabalho em Demasia.

O modelo unidimensional da DUWAS-16 + PTD apresentou índices de ajuste adequados, os itens apresentaram carga fatorial satisfatória no fator geral (Ver Tabela 1). O Modelo 2 avaliou a estrutura de três fatores de primeira ordem para a DUWAS-16 + PTD, com os itens carregando nas respectivas dimensões teóricas, e apresentou índices de ajuste satisfatórios. Em ambos os modelos, os itens 1 (Percepção de Trabalho em Demasia) e 2 (Trabalhar Compulsivamente) apresentam carga fatorial inferior ao esperado $(>0,30)$ (Ver Tabela 1). No Modelo 2 , foi observado que as três dimensões apresentavam relações positivas de alta magnitude (TE e TC, $r=0,93$, $p<0,001$; TC e PTD, $r=0,99, p<0,001$; e TE e PTD, $r=0,89, p<0,001)$. Os achados do Modelo 1 e Modelo 2 sugerem que a dimensão de Percepção de Trabalho em Demasia apresenta uma contribuição baixa na explicação do fenômeno da adição ao trabalho.

A estrutura unidimensional foi indicada como a mais representativa da DUWAS-16 segundo o método de análise paralela. Já os pressupostos teóricos sugerem que a DUWAS-16 deve apresentar dois fatores de primeira ordem. Dessa forma, dois modelos foram avaliados para a DUWAS-16 (Schaufeli et al., 2009). A estrutura unidimensional foi avaliada no Modelo 1, com os 16 itens carregando em um fator geral de adição ao trabalho. A estrutura de dois fatores de primeira ordem foi testada no Modelo 2, de modo que os itens carregavam nas dimensões Trabalhar Compulsivamente e Trabalhar Excessivamente.
Os resultados do Modelo 1 demonstram que, na versão de 16 itens da DUWAS, os índices de ajuste são adequados e os itens apresentam cargas fatoriais satisfatórias para a estrutura unidimensional (Ver Tabela 1). Os índices de ajuste do Modelo 2 também foram adequados, os itens apresentaram cargas fatoriais satisfatórias (Ver Tabela 1) e as dimensões TE e TC estiveram positivamente relacionadas $(r=0,90 ; p<0,001)$.

A estrutura da DUWAS-10 também foi investigada pelo método de análise paralela e pressupostos teóricos da escala. A estrutura unidimensional foi indicada como a mais representativa para a DUWAS-10 pela análise paralela. Dessa forma, foram testados dois modelos para a DUWAS-10, sendo o Modelo 1 unidimensional, de acordo com a análise paralela, e o Modelo 2 de dois fatores de primeira ordem, conforme os pressupostos teóricos. o modelo 1 avaliou a estrutura unidimensional, com os 10 itens carregando em um fator geral de adição ao trabalho. O Modelo 2 investigou a estrutura de dois fatores de primeira ordem, no qual os itens carregaram nas dimensões Trabalhar Compulsivamente e Trabalhar Excessivamente. O modelo unidimensional apresentou índices de ajuste adequados, os itens apresentaram carga fatorial satisfatória no fator geral (Ver Tabela 2). O segundo modelo, o qual avaliou a estrutura de dois fatores de primeira ordem para a DUWAS-10, com os 10 itens carregando em suas respectivas dimensões teóricas, sendo que as dimensões poderiam estar correlacionadas, apresentou índices de ajuste satisfatórios. Como esperado, as dimensões Trabalhar Compulsivamente e Trabalhar Excessivamente estiveram positivamente associadas $(r=0,90 ; p<0,001)$ (Ver Tabela 1).

Tabela 1

Análise Fatorial Confirmatória da DUWAS-16 + PTD (Unidimensional e Três Fatores de Primeira Ordem), DUWAS-16 (Unidimensional e Dois Fatores de Primeira Ordem) e DUWAS-10 (Unidimensional e Dois Fatores de Primeira Ordem)

\begin{tabular}{|c|c|c|c|c|c|c|c|c|c|c|}
\hline \multirow{3}{*}{ Itens } & \multirow{3}{*}{$\begin{array}{c}\text { Modelo } 1 \\
\text { DUWAS-16 } \\
+ \text { PTD } \\
\text { CF } \\
\text { Adição }\end{array}$} & \multirow{2}{*}{\multicolumn{3}{|c|}{$\begin{array}{c}\text { Modelo } 2 \\
\text { DUWAS-16 } \\
+ \text { PTD } \\
\text { CF/F }\end{array}$}} & \multirow{3}{*}{$\begin{array}{c}\text { Modelo } 1 \\
\text { DUWAS-16 } \\
\text { CF } \\
\text { Adição }\end{array}$} & \multirow{2}{*}{\multicolumn{2}{|c|}{$\begin{array}{c}\text { Modelo } 2 \\
\text { DUWAS-16 } \\
\text { CF /F }\end{array}$}} & \multirow{3}{*}{$\begin{array}{c}\text { Modelo } 1 \\
\text { DUWAS-10 } \\
\text { CF } \\
\text { Adição }\end{array}$} & \multirow{2}{*}{\multicolumn{2}{|c|}{$\begin{array}{c}\text { Modelo } 2 \\
\text { DUWAS-10 } \\
\text { CF /F }\end{array}$}} \\
\hline & & & & & & & & & & \\
\hline & & $\mathrm{TE}$ & TC & PTD & & $\mathrm{TE}$ & TC & & $\mathrm{TE}$ & TC \\
\hline 1. Eu não gosto de ter excesso de trabalho. & $0,13^{*}$ & & & $0,15^{*}$ & & & & & & \\
\hline $\begin{array}{l}\text { 2. Desejo não ser tão comprometido com meu } \\
\text { trabalho. }\end{array}$ & $0,15^{*}$ & & $0,16^{*}$ & & $0,15^{*}$ & & $0,15^{*}$ & & & \\
\hline $\begin{array}{l}\text { 3. Eu pareço estar com pressa e correndo } \\
\text { contra o relógio. }\end{array}$ & $0,58^{*}$ & $0,59^{*}$ & & & $0,57^{*}$ & $0,59^{*}$ & & $0,58^{*}$ & $0,61^{*}$ & \\
\hline $\begin{array}{l}\text { 4. Eu continuo trabalhando mesmo quando } \\
\text { meus colegas de trabalho já encerraram } \\
\text { suas atividades. }\end{array}$ & $0,62^{*}$ & $0,64^{*}$ & & & $0,62^{*}$ & $0,64^{*}$ & & $0,62^{*}$ & $0,66^{*}$ & \\
\hline $\begin{array}{l}\text { 5. Trabalhar intensamente é importante para } \\
\text { mim, mesmo quando não gosto do que } \\
\text { estou fazendo. }\end{array}$ & $0,50^{*}$ & & $0,51^{*}$ & & $0,52^{*}$ & & $0,53^{*}$ & $0,53^{*}$ & & $0,55^{*}$ \\
\hline $\begin{array}{l}\text { 6. Mantenho-me ocupado no trabalho, posso } \\
\text { garantir que tenho mais opções disponíveis } \\
\text { para realizar minhas atividades. }\end{array}$ & $0,37^{*}$ & $0,38^{*}$ & & & $0,37^{*}$ & $0,39^{*}$ & & $0,36^{*}$ & $0,37^{*}$ & \\
\hline
\end{tabular}


Tabela 1 (continuação)

Análise Fatorial Confirmatória da DUWAS-16 + PTD (Unidimensional e Três Fatores de Primeira Ordem), DUWAS-16 (Unidimensional e Dois Fatores de Primeira Ordem) e DUWAS-10 (Unidimensional e Dois Fatores de Primeira Ordem)

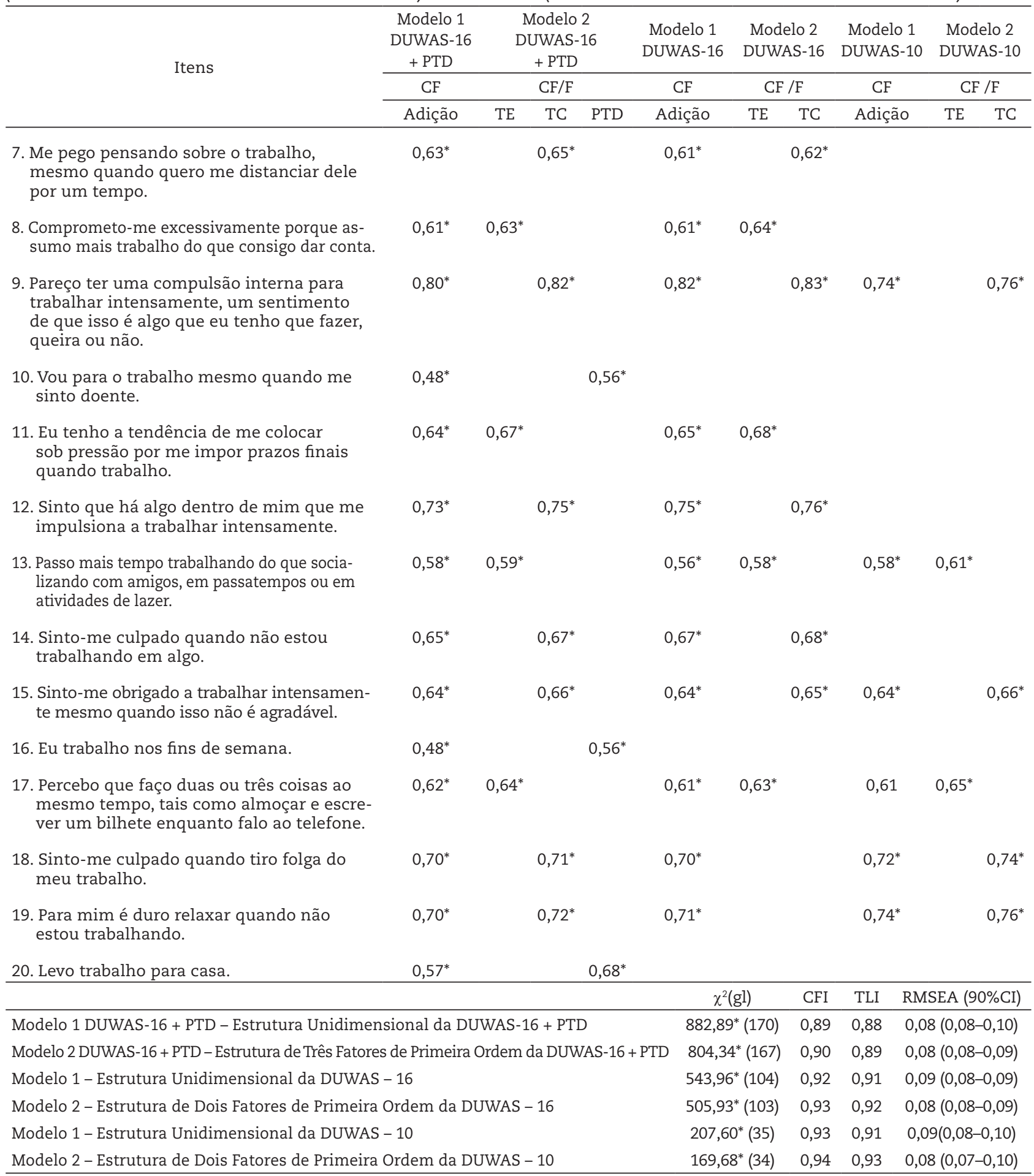

Nota. ${ }^{*} p<0,05, n=571 ; \mathrm{CF}=$ Carga Fatorial do Item; $\mathrm{F}=$ Fator $\mathrm{TC}=$ Trabalhar Compulsivamente, TE=Trabalhar Excessivamente, PTD=Percepção de Trabalho em Demasia

Os índices de ajuste do modelo de dois fatores $\left(\chi^{2}(g l)=169,68(34) ; p<0,001 ; \mathrm{CFI}=0,93 ; \mathrm{TLI}=0,91\right)$ e unifatorial $\left(\chi^{2}(g l)=207,60(35) ; p<0,001 ; \mathrm{CFI}=0,94\right.$; TLI $=0,93)$ da DUWAS-10 são superiores aos modelos de dois fatores $\left(\chi^{2}(g l)=505,93(103) ; p<0,001\right.$; CFI $=0,93$; TLI $=0,92)$ e unifatorial $\left(\chi^{2}(g l)=543,96(104) ; p<0,001\right.$; $\mathrm{CFI}=0,92 ; \mathrm{TLI}=0,91)$ da DUWAS-16, com exceção dos valores de resíduos (RMSEA) que foram semelhantes. 
Observa-se que a DUWAS-10 mensura de forma superior à DUWAS-16 os traços latentes de Trabalhar Excessivamente e Trabalhar Compulsivamente. Trabalhar Excessivamente da DUWAS-10 apresenta superioridade de $8 \%\left(r=0,96 ; p<0,001 ; r^{2}=0,92\right)$ em relação à DUWAS-20. A DUWAS-10 é $12 \% \quad(r=0,94$; $\left.\mathrm{p}<0,001 ; \mathrm{r}^{2}=0,88\right)$ superior na dimensão Trabalhar Compulsivamente. Na dimensão de Adição como um construto unidimensional a DUWAS-10 apresenta superioridade de $8 \%\left(r=0,96 ; p<0,001 ; r^{2}=0,92\right)$ em relação à DUWAS-16.

Os resultados demonstram que a DUWAS-10 é um instrumento adequado para avaliar as dimensões Trabalhar Excessivamente, Trabalhar Compulsivamente e a dimensão geral de Adição ao Trabalho. Devido aos índices de ajuste superiores, da superioridade na mensuração dos traços latentes e da brevidade da DUWAS-10 recomenda-se o uso da versão reduzida da escala em detrimento ao uso da DUWAS-16.

Os índices de confiabilidade das escalas foram satisfatórios. Os índices de confiabilidade, medidos pelo coeficiente de fidedignidade composta $(f c)$, foram adequados tanto para a DUWAS-16 (Trabalhar Compulsivamente, $f_{c}=0,59$; Trabalhar Excessivamente, $f_{c}=0,42$; e Adição ao Trabalho, $f_{c}=0,50$ ), como para a DUWAS-10 (Trabalhar Excessivamente, $f_{c}=0,48$; Trabalhar Compulsivamente, $f_{c}=0,70$; e Adição ao Trabalho, $f_{c}=0,52$ ).
Os resultados das análises de comparação de médias demonstraram que não há diferenças nos índices de Trabalhar Compulsivamente, Trabalhar Excessivamente e Adição ao Trabalho segundo o sexo (homens e mulheres), escolaridade (técnicos e especialistas) e função (gestores e colaboradores). Na Tabela 2, pode ser observado que as médias entre os diferentes grupos se assemelham, tanto que não foi observada a significância estatística e o tamanho de efeito observado foi de baixa magnitude.

Por meio da análise dos percentis dos escores dos participantes, observa-se que 167 profissionais podem estar vivenciando um estado de workaholism. Esses resultados são iguais ao se utilizar a DUWAS-16, ou aplicar a DUWAS-10. Ao investigar o conjunto das dimensões Trabalhar Excessivamente e Trabalhar Compulsivamente foi observado que 95 trabalhadores podem ser classificados como workaholics, tanto com a DUWAS-16, como a DUWAS-10. Além disso, foi observado que 156 profissionais apresentavam índices de Trabalhar Excessivamente superiores ao percentil 75, tanto utilizando a DUWAS-16, como a DUWAS-10. Ao analisar os escores na dimensão Trabalhar Compulsivamente, 162 trabalhadores obtiveram escores superiores ao percentil 75 ao responder a DUWAS-16. Na aplicação da DUWAS-10, 173 participantes obtiveram escores acima do percentil 75 na dimensão Trabalhar Compulsivamente.

Tabela 2

Diferenças das Médias das Dimensões de Trabalhar Excessivamente, Trabalhar Compulsivamente e Adição da Versão Brasileira da DUWAS-16 e DUWAS10 para Sexo, Escolaridade e Função $(n=571)$

\begin{tabular}{|c|c|c|c|c|c|c|}
\hline & \multicolumn{6}{|c|}{ Trabalhar Excessivamente } \\
\hline & $\begin{array}{c}\text { TE-16 } \\
(\mathrm{M}(\mathrm{DP}))\end{array}$ & $t(g l)$ & $d$ & $\begin{array}{c}\text { TE-10 } \\
(\mathrm{M}(\mathrm{DP}))\end{array}$ & $t(g l)$ & $d$ \\
\hline \multicolumn{7}{|l|}{ Sexo } \\
\hline Masculino & $2,3(0,6)$ & \multirow{2}{*}{$-2,7(569)$} & \multirow{2}{*}{0,3} & $2,3(0,6)$ & \multirow{2}{*}{$-2,9(569)$} & \multirow{2}{*}{0,3} \\
\hline Feminino & $2,4(0,6)$ & & & $2,4(0,6)$ & & \\
\hline \multicolumn{7}{|l|}{ Escolaridade } \\
\hline Técnicos & $2,3(0,6)$ & \multirow{2}{*}{$-2,8(569)$} & \multirow{2}{*}{0,2} & $2,3(0,7)$ & \multirow{2}{*}{$-3,1(569)$} & \multirow{2}{*}{0,3} \\
\hline Especialistas & $2,4(0,6)$ & & & $2,5(0,6)$ & & \\
\hline \multicolumn{7}{|l|}{ Função } \\
\hline Colaboradores & $2,3(0,6)$ & \multirow{2}{*}{$-2,8(569)$} & \multirow{2}{*}{0,3} & $2,4(0,6)$ & \multirow{2}{*}{$-3,0(569)$} & \multirow{2}{*}{0,3} \\
\hline \multirow[t]{3}{*}{ Gestores } & $2,5(0,6)$ & & & $2,6(0,7)$ & & \\
\hline & \multicolumn{6}{|c|}{ Trabalhar Compulsivamente } \\
\hline & $\begin{array}{l}\text { TC-16 } \\
(\mathrm{M}(\mathrm{DP}))\end{array}$ & $t(g l)$ & $d$ & $\begin{array}{c}\text { TC-10 } \\
(\mathrm{M}(\mathrm{DP}))\end{array}$ & $t(g l)$ & $d$ \\
\hline \multicolumn{7}{|l|}{ Sexo } \\
\hline Masculino & $1,9(0,5)$ & \multirow{2}{*}{$-1,5(569)$} & \multirow{2}{*}{0,2} & $1,7(0,6)$ & \multirow{2}{*}{$-1,5(569)$} & \multirow{2}{*}{0,1} \\
\hline Feminino & $2,0(0,6)$ & & & $1,8(0,7)$ & & \\
\hline \multicolumn{7}{|l|}{ Escolaridade } \\
\hline Técnicos & $1,9(0,6)$ & \multirow{2}{*}{$-2,6(569)$} & \multirow{2}{*}{0,3} & $1,8(0,6)$ & \multirow{2}{*}{$-1,5(569)$} & \multirow{2}{*}{0,1} \\
\hline Especialistas & $2,0(0,6)$ & & & $1,9(0,6)$ & & \\
\hline
\end{tabular}


Tabela 2 (continuação)

Diferenças das Médias das Dimensões de Trabalhar Excessivamente, Trabalhar Compulsivamente e Adição da Versão Brasileira da DUWAS-16 e DUWAS10 para Sexo, Escolaridade e Função (n=571)

\begin{tabular}{|c|c|c|c|c|c|c|}
\hline & \multicolumn{6}{|c|}{ Trabalhar Compulsivamente } \\
\hline & $\begin{array}{c}\text { TC-16 } \\
(\mathrm{M}(\mathrm{DP}))\end{array}$ & $\mathrm{t}(\mathrm{gl})$ & $d$ & $\begin{array}{c}\text { TC-10 } \\
(\mathrm{M}(\mathrm{DP}))\end{array}$ & $t(g l)$ & $d$ \\
\hline \multicolumn{7}{|l|}{ Função } \\
\hline Colaboradores & $1,9(0,6)$ & \multirow{2}{*}{$-1,8(569)$} & \multirow{2}{*}{0,2} & $1,8(0,6)$ & \multirow{2}{*}{$-1,7(569)$} & \multirow{2}{*}{0,7} \\
\hline \multirow[t]{3}{*}{ Gestores } & $2,0(0,6)$ & & & $2,2(0,6)$ & & \\
\hline & \multicolumn{6}{|c|}{ Adição } \\
\hline & $\begin{array}{l}\text { Adi-16 } \\
(\mathrm{M}(\mathrm{DP}))\end{array}$ & $t(g l)$ & $d$ & $\begin{array}{c}\text { Adi-10 } \\
(\mathrm{M}(\mathrm{DP}))\end{array}$ & $\mathrm{t}(\mathrm{gl})$ & $d$ \\
\hline \multicolumn{7}{|l|}{ Sexo } \\
\hline Masculino & $2,1(0,6)$ & \multirow{2}{*}{$-2,2(569)$} & \multirow{2}{*}{0,1} & $2,1(0,6)$ & \multirow{2}{*}{$-2,5(569)$} & \multirow{2}{*}{0,2} \\
\hline Feminino & $2,2(0,5)$ & & & $2,2(0,5)$ & & \\
\hline \multicolumn{7}{|l|}{ Escolaridade } \\
\hline Técnicos & $2,1(0,5)$ & \multirow{2}{*}{$-4,4(569)$} & \multirow{2}{*}{0,2} & $2,0(0,6)$ & \multirow{2}{*}{$-4,4(569)$} & \multirow{2}{*}{0,3} \\
\hline Especialistas & $2,2(0,5)$ & & & $2,2(0,6)$ & & \\
\hline \multicolumn{7}{|l|}{ Função } \\
\hline Colaboradores & $2,1(0,5)$ & \multirow{2}{*}{$-3,0(569)$} & \multirow{2}{*}{0,2} & $2,1(0,5)$ & \multirow{2}{*}{$-3,2(569)$} & \multirow{2}{*}{0,2} \\
\hline Gestores & $2,2(0,6)$ & & & $2,2(0,6)$ & & \\
\hline
\end{tabular}

Nota. ${ }^{*}<0,001$.

\section{Discussão}

O objetivo deste estudo foi adaptar e investigar as evidências de validade da DUWAS-16 + PTD, DUWAS-16 e a DUWAS-10 para o contexto brasileiro. A DUWAS-16 + PTD apresentou índices de ajuste insatisfatórios, indicando que a PTD não se constitui como uma dimensão central na avaliação do workaholism no contexto brasileiro. As versões DUWAS-16 e DUWAS-10 brasileira apresentaram evidências de validade adequadas para seu uso no país. Entre as contribuições deste estudo, destaca-se a validação de uma escala capaz de mensurar adequadamente o construto da Adição ao Trabalho e a possibilidade de investigar esse fenômeno por meio de uma escala reduzida (DUWAS-10).

Os dados apontam para a importância de compreender de modo mais aprofundado aspectos relacionados às possíveis diferenças culturais nos achados. Diferentemente do proposto por Castillo e Gómez (2012), a dimensão Percepção de Trabalho em Demasia (PTD) não contribui na explicação do fenômeno da adição ao trabalho no contexto brasileiro. Dessa forma, foram propostas a versão DUWAS-16 e DUWAS-10 para investigar as dimensões Trabalhar Compulsivamente e Trabalhar Excessivamente, dimensões centrais do workaholism, e a adição ao trabalho enquanto um fenômeno unidimensional (Schaufeli et al., 2009).

De modo geral, os resultados da presente pesquisa demonstram evidências de validade de conteúdo e de construto para uso da DUWAS no Brasil. O estudo evidenciou também que o fator Trabalhar
Compulsivamente (TC) apresenta relação de alta magnitude com o fator Trabalhar Excessivamente (TE), pois estas são as dimensões centrais na adição ao trabalho. Por outro lado, os achados revelam uma estrutura fatorial coerente e apoiada no modelo teórico de Adição ao Trabalho, proposto pelos autores na escala original (Hu et al., 2014; Mazzetti et al., 2016; Schaufeli et al., 2009). Pelo exposto, pode-se concluir que a versão brasileira do instrumento apresenta a estrutura esperada em termos de consistência interna e validade fatorial, estimulando seu uso na avaliação da adição ao trabalho na realidade brasileira.

Foi observada que a DUWAS-10 apresentou índices de ajuste e capacidade de explicação das dimensões Trabalhar Excessivamente, Trabalhar Compulsivamente e Adição ao Trabalho superiores aos índices da DUWAS-16. Dessa forma, considerando a importância de serem utilizados instrumentos breves, recomenda-se o uso da DUWAS-10. Além disso, sugere-se que estudos que objetivam investigar as relações do workaholism com outras variáveis devem analisar os dados, utilizando, preferencialmente, a solução unifatorial, tendo em vista a correlação de alta magnitude entre as dimensões Trabalhar Excessivamente e Trabalhar Compulsivamente.

Os resultados demonstraram, ao analisar os resultados do escore de Adição ao Trabalho (Schaufeli et al., 2009), que 29\% da amostra investigada pode estar vivenciado um estado de workaholism. Investigando a presença de workaholism pela combinação dos escores das dimensões Trabalhar Excessivamente e Trabalhar Compulsivamente, nota-se que $17 \%$ dos profissionais 
podem ser categorizados como workaholics (Schaufeli et al., 2009). Além disso, entre $27 \%$ a $30 \%$ dos participantes investigados apresentaram altos índices nas dimensões Trabalhar Excessivamente e Trabalhar Compulsivamente. Esses achados demonstraram que os escores de Adição ao Trabalho podem ser utilizados para um screening para identificar os profissionais workaholics. Já a análise por meio das dimensões Trabalhar Excessivamente e Trabalhar Compulsivamente devem ser usadas para análises em profundidade dos profissionais identificados como workaholics.

O fator Percepção de Trabalho em Demasia (PTD) apresentou resultados que merecem uma discussão mais aprofundada. Ressalta-se que, apesar do item 1 ter carregado menos que os demais, não houve alteração das características psicométricas da escala. Esse baixo resultado na carga fatorial do item 1 (Eu não gosto de ter excesso de trabalho) pode representar diferenças culturais. Ao avaliar esse item qualitativamente, sugere-se que os participantes podem ter sido influenciados pela desejabilidade social, o que os levaria a responder considerando qual a resposta mais aceitável ou aprovada socialmente e tenderia a negar sua associação pessoal com opiniões e comportamentos que seriam desaprovados socialmente (Pacico, 2015). Esse é um tipo de comportamento que pode variar de cultura para cultura, pois algumas respostas são consideradas mais desejáveis do que outras em certos contextos, quando comparadas. Da mesma forma, opiniões, valores e atitudes também estão sujeitos às normas e sanções sociais.

Quanto à análise dos dados sociodemográficos, não foram observadas diferenças de acordo com o sexo, escolaridade e função dos profissionais. Esses achados demonstram que profissionais com diferentes características sociodemográficas podem estar expostos ao desenvolvimento do workaholism, uma vez que essa síndrome está mais relacionada às características de compulsão dos indivíduos (Hu et al., 2014; Mazetti et al., 2014) do que as suas características sociodemográficas.

Foram limitações deste estudo a amostra predominantemente do sexo feminino e com concentração nas regiões Sul e Sudeste do Brasil. O que sugere que novos estudos devem ser realizados para verificação das normas para a população brasileira, além de pesquisas para testar a validade discriminante e a estabilidade fatorial da versão brasileira da DUWAS.

Ademais, sugere-se que o fator Percepção de Trabalho em Demasia (PTD) deve ser aprofundado em estudos que visem compreender o significado desse construto para a cultura brasileira. Ressalta-se que a sociedade contemporânea valoriza e reforça socialmente o comportamento de adição ao trabalho. Algumas culturas corporativas estimulam e privilegiam as condutas que ultrapassam o contrato funcional de trabalho e os horários oficiais estabelecidos. Há que se considerar que conotações positivas de fluxo de trabalho árduo na cultura brasileira podem estar reforçando padrões de comportamento adicto nesse contexto de modo diferenciado para o Brasil. Portanto, sendo socialmente aceitável trabalhar em demasia, o modo como os brasileiros compreendem o quê seria um excesso está diretamente ligado ao sentido que dão ao trabalho na vida social.

\section{Referências}

American Educational Research Association, American Psychological Association, \& National Council on Measurement in Education. (2014). Standards for educational and psychological testing. Washington, DC: American Psychological Association.

Brown, T. A. (2006). Confirmatory factor analysis for applied research. New York: The Guilford Press.

Carlotto, M. S. (2011) Adição ao trabalho e relação com fatores de risco sociodemográficos, laborais e psicossociais. Psico-USP, 16(1), 87-95. doi: 10.1590/S1413-82712011000100010

Carlotto, M. S., Wendt, G. W., Lisboa, C., \& Moraes, M. A. (2014). Preditores da adição ao trabalho em trabalhadores que utilizam tecnologias de informação e comunicação. Trends in Psychology, 22(2) 377-387. doi: 10.9788/TP2014.2-09

Castillo, J. A., \& Gómez M. C. (2012). Excessive work or addiction to work: Workaholism in a Colombian company. A Pilot study trough application of DUWAS test. Revista Ciencias de la Salud, 10(3), 307-322. Recuperado de http://www.scielo.org.co/scielo.php?script=sci_ arttext\&pid $=$ S1692-72732012000300002

Hayton, J. C., Allen, D. G., \& Scarpello, V. (2004). Factor retention decisions in exploratory factor analysis: A tutorial on parallel analysis. Organizational Research Methods, 7(2), 191-205. doi: 10.1177/1094428104263675

Hu, Q., Schaufeli, W. B., Taris, T. W., van Hessen, D. J., Hakanen, J. Salanova, M., \& Shimazu, A. (2014). "East is East and West is West and never the twain shall meet" Work engagement and workaholism across Eastern and Western cultures. Journal Behavioral and Social Science, 1, 6-24. Recuperado de https://dspace.library.uu.nl/handle/1874/305458

Killinger, B. (1991) Workaholic: The respectable addicts. New York: Simon \& Schuster.

Mazzetti, G., Biolcati, R., Guglielmi, D., Vallesi, C., \& Schaufeli, W. (2016). Individual characteristics influencing physicians’ perceptions of job demands and resources: The role of affectivity, work engagement and workaholism. International Journal of Environmental Research and Public Health, 13, 567-587. doi: 10.3390/ijerph13060567

Mazetti, G., Schaufeli, W. B., \& Guglielmi, D. (2014). Are workaholics born or made? Relations of workaholism with person characteristics and overwork climate. International Journal of Stress Management, 21, 227-254. doi: 10.1037/a0035700

Muthén, L. K., \& Muthén, B. O. (2010). Mplus: Statistical analysis with latent variables. User’s guide. Los Angeles: Muthén \& Muthén. 
Pacico, J. C. (2015). Como é feito um teste? Produção de itens. Em C. S. Hutz, D. R. Bandeira \& C.M. Trentini, (Orgs.). Psicometria (pp. 55-70). Porto Alegre: Artmed.

Schaufeli, W. B., Taris, T. W., \& Bakker, A. (2008). It takes two Tango: Workaholism is working excessively and working compulsively. Em R. J. Burke \& C.L. Cooper, (Eds.). The long work hours culture. Causes, consequences and choices (pp. 203-206). UK: Emerald.

Schaufeli, W. B., Shimazu, A, Taris, T. W. (2009). Being driven to work excessively hard. The evaluation of a two-factor measure of workaholism in the Netherlands and Japan. Cross-Cultural Research, 43, 320-348. doi: 10.1177/1069397109337239

Shimazu, A., Schaufeli, W. B., Kamiyama, L., \& Kawakami, N. (2015). Workaholism vs. work engagement: The two different predictors of future well-being and performance. International Journal of Behavioral Medicine, 22, 18-23. doi: 10.1007/s12529-014-9410-x

Sousa, A. P., Mónico, L. S., \& Castro, F. V. (2012). Workaholic ou worklover: A importância da inteligência emocional. International Journal of Developmental and Educational Psychology, 4(1), 281-289.

\section{Sobre os autores}

Ana Claudia Souza Vazquez é psicóloga, com doutorado em Administração na UFRGS, professora da Universidade Federal de Ciências da Saúde de Porto Alegre e presidente da Associação Brasileira de Psicologia Positiva.

Clarissa Pinto Pizarro de Freitas é psicóloga, Mestre e Doutora em Psicologia; docente do Pós-Graduação de Psicologia Social da Universidade Salgado de Oliveira.

Andressa Cyrre é psicóloga, Pós-graduanda de Gestão de Pessoas no Centro Universitário Ritter dos Reis, Analista de RH na ABRHRS.

Claudio Simon Hutz é psicólogo, com doutorado na Universidade de Iowa (USA), professor titular na Universidade Federal do Rio Grande do Sul e pesquisador 1A do CNPq.

Wilmar B. Schaufeli é psicólogo, professor pesquisador na Leuven University (Bélgica), professor titular em Psicologia Organizacional e do trabalho na Utrecht University (Holanda) e sócio da empresa de consultoria Triple i Human Capital. 